\title{
A NEW MODEL OF INHOMOGENEOUS DEFORMATION USED FOR CRAZE FORMATION
}

\author{
S. Kreitmeier*, D. Göritz \\ Universität Regensburg, Institut für Physik III, Universitätsstr. 31, \\ D-8400 Regensburg, Germany
}

\begin{abstract}
The coil-strand (CS) model is described. After a phase of elastic deformation of an isotropic coil a highly oriented strand and a still isotropic rest coil are formed. Entropy and several energy contributions are calculated and compared with experimental data. The constant tensile stress acting on the craze surface, the inverse relationship between fibril extension ratio and fibril volume fraction and the temperature dependence of the product of tensile stress and fibril diameter can be calculated directly from the model. Some other properties are discussed.
\end{abstract}

\section{INTRODUCTION}

The coil-strand (CS) model is a new way to describe the deformation behaviour of polymers. It is a simple and interesting approach to the cold drawing phenomena. This will be shown for crazes.

\section{CRAZE MORPHOLOGY}

Before the explanation of the CS model there will be a short listing of the craze morphology. Crazes are microflaws in any polymer materials perpendicular to the draw direction. The crazes are, in contrast to normal cracks, filled with highly strained fibrils along the draw direction. Typical dimensions for air crazes are length $\sim 100 \mu \mathrm{m}$, width $\sim 1-2 \mu \mathrm{m}$, fibril extension ratio $\lambda_{\text {fibril }} \sim 2-5$, fibril volume fraction $\sim 20-50 \%$ and fibril diameter $\sim 5-15 \mathrm{~nm}$. A schematic view is shown in Fig. 1.

\section{EXPERIMENTAL FINDINGS}

Three experimental findings will be mentioned here because the following theory will focus on them.

(a) The tensile stress acting on the craze surface is constant over the whole craze length. The peaks at the beginning and at the crack initiation are due to other mechanisms (Fig. 2) (1). 


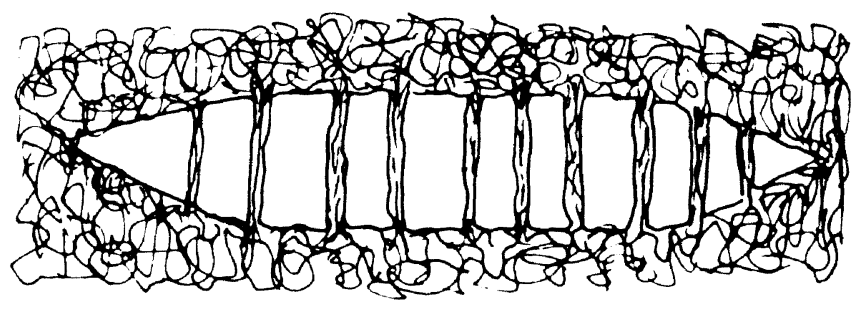

Fig. 1. Schematic view of an air craze

(b) The product of tensile stress and fibril diameter is essentially independent of temperature and draw ratio (Fig. 3).

(c) The volume fraction of the fibrils in a craze is inversely proportional to the fibril extension ratio.

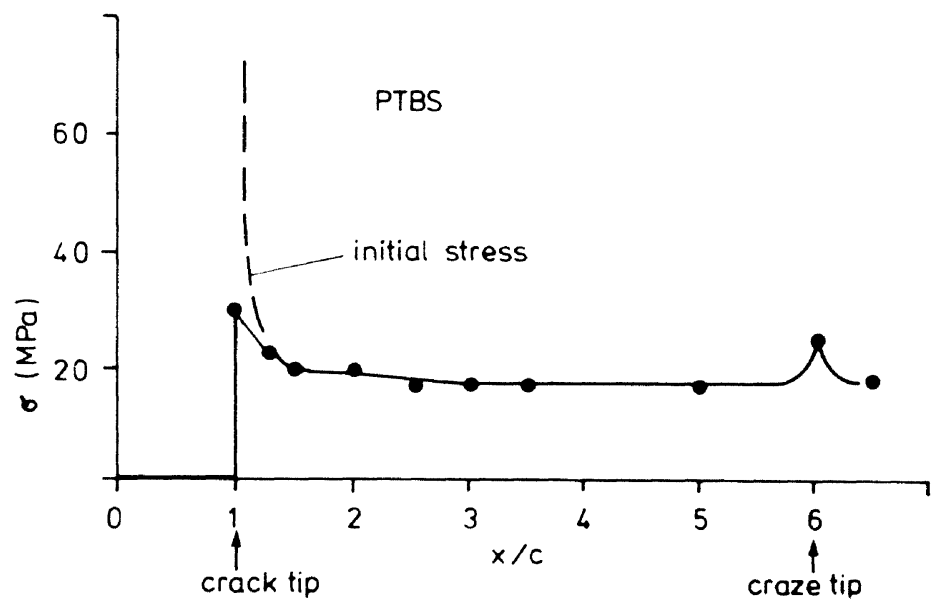

Fig. 2. Tensile stress versus distance $x$, measured in units of half crack length $c$, for poly(tertbutylstyrene) (PTBS)(1)

\section{DEFORMATION MECHANISM}

The well known affine deformation (Flory, Kuhn, Treloar) is a good theory for describing the rubber elasticity, but its essential feature is to be homogeneous. Therefore, it is difficult to explain the formation of highly oriented fibrils according to a process shown in Fig. 4.

In contrast to that the new CS model is an excellent way to describe such a deformation situation. The CS model is totally inhomogeneous. Its essential feature is 


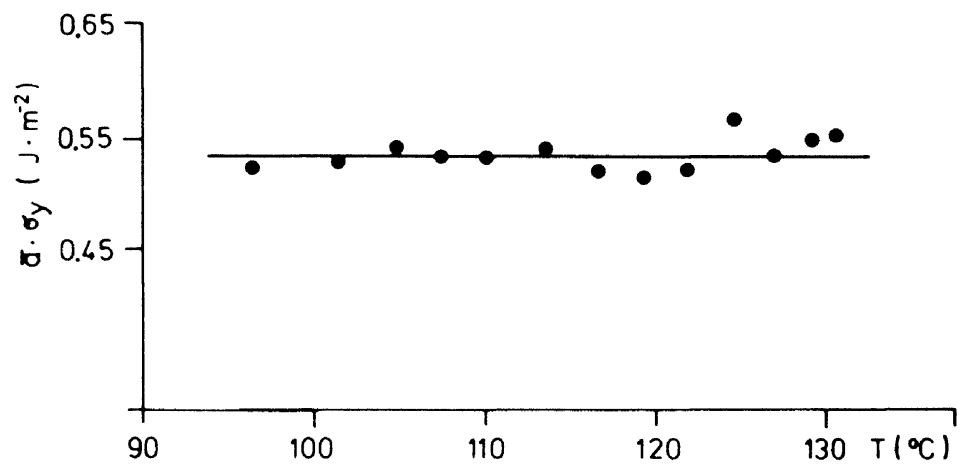

Fig. 3. Product of tensile stress and fibril diameter versus temperature (3)
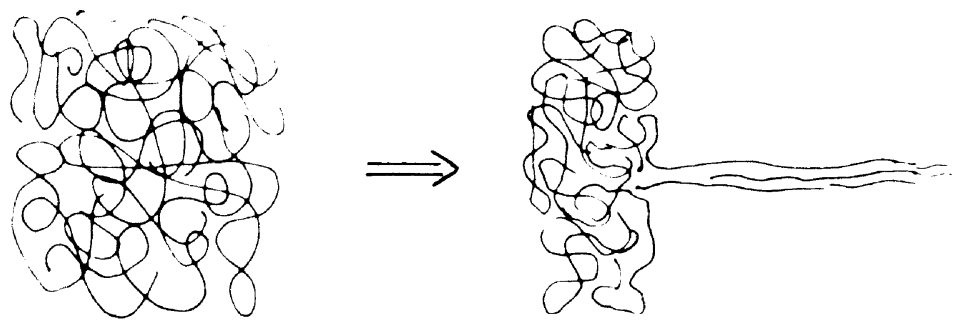

Fig. 4. Inhomogeneous deformation of a coil

that the random polymer coil is not transformed to an ellipse, but, after a phase of elastic (Hookean) stretching, it forms a highly oriented strand without any intermediate state. The rest of the coil remains as isotropic and unoriented as the initial coil. This situation does not change during further deformation. Step by step there will be a transport of subunits from the isotropic rest coil into the highly oriented strand. From the very beginning of the deformation the orientation of the strand is the same. It does not increase although the strand is getting longer and longer. At any time during stretching the difference between the orientation of the rest coil and the strand remains constant.

\section{CS MODEL ASSUMPTIONS}

For calculation purposes the inital coil will be approximated by a cube filled with $N$ statistically arranged subunits (Fig. 5). The subunit may represent a monomer or an ensemble of monomers like the statistical segment in Kuhn's theory. It is assumed 


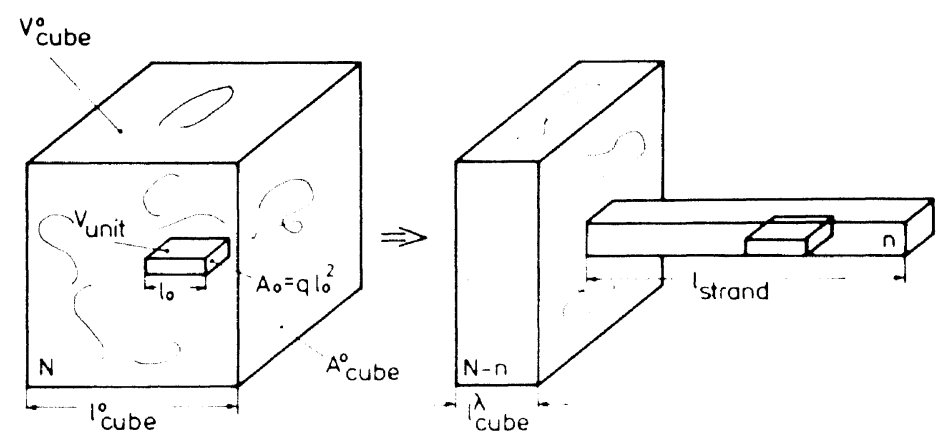
a) $\lambda=1$
b) $\lambda>1$

Fig. 5. The CS model:

deformation unit before and after deformation

$V_{\text {cube }}^{0}$ : volume of the initial cube

$l_{\text {cube }}^{0}$ : length of the initial cube

$A_{\text {cube }}^{0}$ : cross-section of the initial cube

$V_{\text {unit }}$ : volume of a subunit

$l_{0}:$ length of a subunit

$A_{0}$ : cross-section of a subunit

$l_{\text {cube }}^{\lambda}:$ length of the rest cube

$l_{\text {strand }}$ : length of the fibril

$N$ : number of subunits in the initial cube

$n$ : number of subunits in the fibril

that the cube is reduced only in draw direction. The dimensions perpendicular to the draw direction remain unaltered. This means that free volume is created. The developing strand consists of $n$ fully oriented subunits. The rest cube is therefore built up out of $(N-n)$ still statistically arranged subunits.

We call the initial cube and the rest cube and fibril a deformation unit.

The total craze is then built up out of parallel arranged deformation units.

The extension ratio of the total deformation unit is defined as

$$
\lambda_{\text {def }}=\frac{\text { length of fibril }\left(l_{\text {strand }}\right)+\text { length of rest cube }\left(l_{\text {cube }}^{\lambda}\right)}{\text { length of initial cube }\left(l_{\text {cube }}^{0}\right)}
$$

The fibril extension ratio is defined as

$$
\lambda_{\text {fibril }}=\frac{\text { length of fibril }\left(l_{\text {strand }}\right)}{\text { length of initial cube of fibril matter }\left(l_{\text {fibril matter }}^{0}\right)}
$$

When calculating $\lambda_{\text {fibril }}$ there results a constant value independent of $\lambda_{\text {def }}$. The model shows no fibril creep. It represents the now accepted behaviour of air crazes which grow through bulk drawing. 


\section{RESULTS DUE TO GEOMETRY}

The special geometrical behaviour of the model has some interesting consequences. Defining the fibril spacing as the width of the cube $D_{\text {cube }}$, this is the distance between parallel deformation units, the fibril spacing is related to the fibril diameter according to

$$
D_{\text {cube }}=D_{\text {fibril }} \cdot \lambda_{\text {fibril }}^{1 / 2}
$$

For the fibril volume fraction one gets

$$
V_{\text {fibril }}=\frac{1}{\lambda_{\text {fibril }}}
$$

The experimental finding (c) namely the indirect relationship between fibril volume fraction and fibril extension ratio therefore is a natural result of the CS model.

\section{ENERGY AND ENTROPY}

To reach out for the constant tensile stress several energy contribution were evaluated. In special these are surface energy, trans-gauche energy, elastic energy and chain scission. In addition the entropy was considered, too. Due to the constant orientation difference between the rest cube and the fibril all energies show a common behaviour:

$$
E^{\mathrm{CS}}=N \cdot \Delta E\left(\frac{\lambda_{\text {def }}-1}{\lambda_{\text {fibril }}-1}\right)
$$

$\Delta E$ is the constant energy change which one subunit experiences when crossing over from the rest cube to the fibril.

The same is valid for the entropy:

$$
S^{\mathrm{CS}}=-N \Delta S\left(\frac{\lambda_{\mathrm{def}}-1}{\lambda_{\text {fibril }}-1}\right)
$$

$\Delta S$ is the constant entropy loss of one subunit.

\section{CONSTANT TENSILE STRESS}

With the tensile stress being

$$
\sigma_{\mathrm{n}} d \lambda_{\mathrm{def}}=d E-T d S \quad \text { per volume }
$$

there results

$$
\begin{array}{r}
\sigma_{\mathrm{n}}=\left[\left(T N / V_{\text {cube }}^{0}\right) \cdot \Delta S+\left(N / V_{\text {cube }}^{0}\right) \cdot \Delta E+\right. \\
\left.\left(4 \gamma / V_{\text {cube }}^{0}\right) \cdot A_{\text {fibril }} \lambda_{\text {fibril }}^{3 / 2}\right] \cdot \frac{1}{\lambda_{\text {fibril }}-1}
\end{array}
$$


$\gamma$ describes the surface energy.

The volume of the initial cube, $V_{\text {cube }}^{0}$, the cross section of the fibril, $A_{\text {fibril }}$ and the number $N$ as well as the entropy and energy changes do not depend on $\lambda_{\text {def }}$. Therefore, the total formula is independent of $\lambda_{\text {def }}$. Thus, the tensile stress is constant explaining the experimental finding (a).

The overall reason is the constant difference between the properties of the rest coil and the strand. From that the linear energy and entropy law arises.

\section{THE PRODUCT OF TENSILE STRESS AND DIAMETER}

The product of tensile stress and fibril diameter was evaluated and compared with an experimental value gained for polystyrene (4).

$$
\begin{aligned}
\sigma_{\mathrm{n}} \cdot D_{\text {fibril }}= & k_{\mathrm{B}} T \ln z \cdot \frac{D_{\text {fibril }} / V_{\text {unit }}}{\lambda_{\text {fibril }}-1} \\
& +G^{0} \frac{\left(\lambda_{\text {iso }}-1\right)^{2}}{\lambda_{\text {iso }}}\left(\frac{\lambda_{\text {fibril }} \lambda_{\text {iso }}}{\lambda_{\text {iso }}-1+\frac{G^{\lambda}}{G^{0}}}-1\right) \frac{D_{\text {fibril }}}{\lambda_{\text {fibril }}-1} \\
& +\frac{\Delta E_{\mathrm{gt}} D_{\text {fibril }} / V_{\text {unit }}}{\lambda_{\text {fibril }}-1} \\
& +\frac{E_{\text {cov }} \sqrt{M_{0}} \nu_{\mathrm{e}}}{4 \rho N_{\mathrm{A}} k} \cdot \frac{A_{\text {cube }}}{V_{\text {unit }}} \cdot \frac{\lambda_{\text {fibril }}^{-1}}{\lambda_{\text {fibril }}^{1 / 2}+1} \\
& +\frac{4 \gamma(T)}{\lambda_{\text {fibril }}-1}
\end{aligned}
$$

The calculated values are shown in Table 1.

The first term is the entropy contribution. $k_{\mathrm{B}}$ is the Boltzmann konstant and $z$ is an effective coordinative number. Its contribution to the product is not significant.

The second term is due to elastic changes. $G^{0}$ and $G^{\lambda}$ are the different Young moduli for the isotropic cube and the highly oriented fibril. $\lambda_{\text {iso }}$ characterizes the elastic strain within the initial cube before the CS formation begins.

The third term results from trans-gauche energy differences, $\Delta E_{\mathrm{gt}}$. The great value in Table 1 depends to a high degree on the assumption that every gauche state transits into a trans state during fibril formation. In reality this is not valid. Therefore, the trans-gauche energy is largely overestimated. Nevertheless, the value shows what it may be. 
Table 1: Experimental and calculated values of $\sigma_{\mathrm{n}} \cdot D_{\text {fibril }}$ for polystyrene

\begin{tabular}{ll}
\hline Experimental value (4): & $(300 \pm 50) \mathrm{mJ} / \mathrm{m}^{2}$ \\
Entropy term & calculated values \\
Elastic energy term & $\sim 16 \mathrm{~mJ} / \mathrm{m}^{2}$ \\
Trans-gauche energy term & $\sim(40-110) \mathrm{mJ} / \mathrm{m}^{2}$ \\
Chain scission term & $\sim(60-110) \mathrm{mJ} / \mathrm{m}^{2}$ \\
Surface energy term & $\sim 110 \mathrm{~mJ} / \mathrm{m}^{2}$ \\
\hline
\end{tabular}

The fourth term is derived from chain scission. $E_{\text {cov }}$ is the covalent bonding energy. $M_{0}$ is the molecular weight of the monomer. $\nu_{\mathrm{e}}$ represents the entanglement density, $\rho$ the density of the polymer. $N_{\mathrm{A}}$ is Avogadro's number and $k$ is a constant with values in the range of $(0.05-0.1) \mathrm{nm} / \sqrt{\mathrm{MW}}$. It was calculated with the assumption that chain scission occurs whenever a subunit must surpass a distance greater than the maximum entanglement distance.

The last term is due to the new surfaces. It corresponds to a formula derived by Paredes, Fischer (3).

Although the Table 1 is only a rough estimate it shows where contributions to the product can be found and that there are enough possibilities to reach the experimental value.

\section{TEMPERATURE DEPENDENCE}

As the last point the temperature dependence of the calculated product will be discussed.

At first the fibril diameter increases with temperature whereas the fibril extension ratio is essentially independent of temperature when using not too great draw ratios. The entropy also increases with temperature. On the other hand, the number of trans-gauche changes, the Young moduli, the surface energy and the rate of chain scission decrease with temperature.

Therefore, the experimental finding (b) of constancy of the product $\sigma_{\mathbf{n}} \cdot D_{\text {fibril }}$ is merely due to compensation effects and it is surely not valid for every polymer and the whole temperature range. A similar argumentation holds for varying draw ratios.

\section{CONCLUSION}

In the present paper we have shown that the CS model is a good description for crazes. The CS model is a model for inhomogenous deformation. Its essential feature is the formation of a highly oriented fibril out of an isotropic and unoriented coil without any intermediate state. The geometric model assumption of linear reduction only in 
the draw direction explains the inverse proportion between fibril volume fraction and fibril extension ratio. The constant orientation difference between rest coil and fibril during deformation gives rise to a linear energy law and thus to a constant tensile stress which is found to act on the craze surface. The calculated product of tensile stress and fibril diameter shows interesting possibilities for the interpretation of the experimental data.

\section{REFERENCES}

(1) Kramer, E.J., Adv. Polym. Sci. 52/53, 2 (1983)

(2) Dettenmaier, M., Adv. Polym. Sci. 52/53, 57 (1983)

(3) Paredes,E. Fischer, E.W., Makromol. Chem. 180, 2707 (1979)

Paredes,E. Fischer, E.W., J. Polym. Sci. 20, 929 (1982)

(4) Berger, L.L., Buckley, D.J., Kramer, E.J., Brown, H.R., Bubeck, R.A., J. Polym. Sci. 25, 1679 (1987)

Berger, L.L., Kramer, E.J., J. Mater. Sci. 23, 3536 (1988)

(5) Wohlrab, J., Dissertation, Universität Regensburg, 1984

(6) Hopfenmüller, M.K., Dissertation, Universität Regensburg, 1986

(7) Kreitmeier, S., Dissertation, Universität Regensburg, 1990

(8) Kreitmeier, S., Göritz, D., Theory of craze formation using an inhomogeneous deformation model, 3rd Lausanne Polymer Meeting, 1989, P52 